\title{
Relações entre a distribuição das espécies de diferentes estratos e as características do solo de uma floresta aluvial no Estado do Paraná, Brasil
}

\author{
Joema Carvalho ${ }^{1,3}$, Márcia Cristina Mendes Marques ${ }^{2,5}$, Carlos Vellozo Roderjan ${ }^{3}$, Murilo Barddal ${ }^{1,3}$ e \\ Silas Garcia Aquino de Sousa ${ }^{4}$
}

Recebido em 7/08/2007. Aceito em 25/02/2008

\begin{abstract}
RESUMO - (Relações entre a distribuição das espécies de diferentes estratos e as características do solo de uma floresta aluvial no Estado do Paraná, Brasil). Entre os fatores que influenciam a distribuição de espécies nas comunidades vegetais localizadas em regiões ribeirinhas, as características do solo são consideradas os mais importantes. No presente estudo, foram avaliadas a composição florística, a diversidade e a estrutura de três estratos da vegetação e suas relações com as características do solo em um fragmento de floresta aluvial na bacia do rio Iguaçu, no estado do Paraná. Buscou-se verificar se a estrutura e a diversidade da floresta nos diferentes estratos podem ser explicadas por fatores edáficos e hídricos. Caracterizaram-se os parâmetros estruturais e a composição florística de três estratos da vegetação \% de regeneração $(20 \mathrm{~cm} \leq$ altura $\leq 1,30 \mathrm{~m} ; 80$ sub-parcelas de $1 \mathrm{~m} \times 1 \mathrm{~m}$ ), intermediário (perímetro à altura do peito - PAP $<15 \mathrm{~cm}$, altura > 1,30 m; 20 sub-parcelas de $5 \mathrm{~m} \times 5 \mathrm{~m}$ ) e superior (PAP $\geq 15 \mathrm{~cm}, 20$ parcelas de $10 \mathrm{~m} \times 10 \mathrm{~m}$ ) - e correlacionaram-se as abundâncias das espécies com os parâmetros do solo e do lençol freático de cada parcela. A floresta caracterizou-se por baixa diversidade $\left(H^{\prime}=2,36\right.$ no estrato de regeneração, 2,49 no estrato no estrato intermediário e 1,59 no estrato superior) e alta similaridade florística entre estratos (índices de similaridade de Sørensen $\geq 0,65$ ). As relações entre a distribuição espacial das espécies e as características do solo foram distintas entre estratos: no estrato superior correlacionou-se com a profundidade do lençol freático e o teor de matéria orgânica; no intermediário, com profundidade do lençol e teor de alumínio, porém no estrato inferior nenhum dos fatores ambientais analisados explicou a distribuição das espécies. Os resultados sugerem que a estrutura e a composição florística destas comunidades aluviais refletem os diferentes níveis de interação ao longo do ciclo de vida dos indivíduos.
\end{abstract}

Palavras-chave: estrato de regeneração, floresta com Araucária, floresta Ombrófila Mista, lençol freático, nutrientes do solo

\begin{abstract}
Species distribution relationships of different strata and soil characteristics in an alluvial forest in Paraná State, Brazil). Soil characteristics are the most important factors influencing species distribution in alluvial forests. Here we evaluated species composition, diversity and structure of three forest strata and their relationship with soil characteristics in a fragment of alluvial forest in the Iguaçu River basin, Paraná state. We asked if forest structure and diversity can be explained by edaphic and hydrological factors. We evaluated structural variables and floristic composition of three forest strata: regeneration stratum $(20 \mathrm{~cm} \leq$ height $\leq 1.30 \mathrm{~m}$; $80 \mathrm{subplots}$, $1 \mathrm{~m} \times 1 \mathrm{~m}$ ), intermediate stratum (perimeter at breast height - pbh $<15 \mathrm{~cm}$, height $>1.30 \mathrm{~m} ; 20$ subplots, $5 \mathrm{~m} \times 5 \mathrm{~m}$ ) and upper stratum (pbh $\geq 15 \mathrm{~cm}, 20$ subplots, $10 \mathrm{~m} \times 10 \mathrm{~m}$ ). In addition, we correlated species abundance with soil parameters in each subplot. The forest was characterized by a low diversity (Shannon's index: regeneration stratum $=2.36$; intermediate stratum $=2.49$; upper stratum $=1.59$ ) and high floristic similarity among strata (Sørensen index $\geq 0.65$ ). The relationship of species spatial distribution and soil characteristics was distinct among strata: species distribution was correlated with soil water-table levels and organic matter content in the upper stratum and with soil water-table levels and aluminum concentration in the intermediate stratum. On the other hand no significant correlation was verified in the regeneration stratum. Our results suggest that community structure and floristic composition in alluvial forests reflect different interactions during individual life cycles.
\end{abstract}

Key words: Araucaria forest, ombrophilous forest, regeneration stratum, soil nutrients, water table

\section{Introdução}

A Floresta Ombrófila Mista (Floresta com Araucária) que ocorre, principalmente, nos estados do sul do Brasil, é caracterizada por apresentar flora de origem tropical (afro-brasileira) e temperada (austro-brasileira) e por diferenciar-se florística e estruturalmente em função da altitude e latitude (Veloso et al. 1991). Nas áreas próximas aos rios, a Floresta Ombrófila Mista percorre, em geral, terrenos recentes (Holoceno) de geomorfologia plana até suave-ondulada e em processo de sedimentação aluvial, onde os solos são, freqüentemente, cobertos por águas

\footnotetext{
Pós-Graduação em Engenharia Florestal

2 Universidade Federal do Paraná, SCB, Departamento de Botânica, C. Postal 19.031, 81531-980 Curitiba, PR, Brasil

3 Universidade Federal do Paraná, SCA, Departamento de Ciências Florestais, 80201-170 Curitiba, PR, Brasil.

4 Empresa Brasileira de Pesquisa Agropecuária, Centro de Pesquisa Agroflorestal da Amazônia Ocidental - CPAA, C. Postal 319, 69010-970 Manaus, AM, Brasil

5 Autor para correspondência: mmarques@ufpr.br
} 
fluviais (Klein \& Hatschbach 1962; Roderjan et al. 2002). Nestes locais, as comunidades florestais apresentam-se diferenciadas, em geral, com baixa riqueza (Klein \& Hatschbach 1962), constituindo as chamadas Florestas Ombrófilas Mistas Aluviais (Veloso et al. 1991).

As comunidades vegetais que ocupam áreas aluviais são influenciadas principalmente pelas características do substrato (Curcio et al. 2007). A dinâmica de movimentação do curso da água promove erosão e deposição de sedimentos, influenciando no relevo aluvial (Troppmair \& Machado 1974; Salo \& Kalliola 1991). Tais variações na altura do terreno, juntamente com a profundidade do lençol freático e a velocidade de drenagem da água (Torezan \& Silveira 2002; Bianchini et al. 2003; Dalanesi et al. 2004) influenciam fortemente a ocorrência de espécies (Joly 1991). Adicionalmente, as características nutricionais do solo são influenciadas pela baixa drenagem do terreno (Dalanesi et al. 2004), o que deve exercer influência no estabelecimento de plantas (Ivanauskas et al. 1997; Kotchetkoff-Henriques et al. 2005), bem como na abundância relativa das espécies (Lima et al. 2003). No entanto, como as características do substrato não são constantes no tempo e no espaço, espécies acumulam-se nos microsítios disponíveis, o que, em longo prazo, favorece uma riqueza de espécies relativamente alta na comunidade aluvial (Bianchini et al. 2003; Lima et al. 2003).

As condições físicas do ambiente influenciam a ocorrência de plantas em todas as fases de sua vida, mas é no estádio juvenil que a sobrevivência é mais crítica (Crawley 1997). No caso de espécies que ocorrem em florestas aluviais, o estresse provocado pela inundação pode tanto limitar o crescimento das plântulas quanto não causar efeitos (Joly 1991; 1996), dependendo do grau de tolerância da espécie (Marques et al. 2003). Como as demandas energéticas variam conforme o desenvolvimento da planta (Medri et al. 2002), é possível que indivíduos de diferentes idades sofram influência diferenciada do excesso de água no solo e das alterações na concentração de macro e micronutrientes.

A partir do estádio juvenil até o adulto, os indivíduos de uma população vegetal passam por uma série de filtros ambientais e biológicos que limitam seu estabelecimento (Connel et al. 1984). Durante este processo, as espécies passam a experimentar formas diferenciadas de interação, resultando em comunidades de estrutura e composição distintas (Richards 1979; Hubbell \& Foster 1990). Espécies raras e abundantes na comunidade podem sofrer efeitos diferenciados durante a passagem por tais filtros. Assim, por exemplo, uma espécie abundante no estádio juvenil pode sofrer mais os efeitos da densidade que uma espécie rara, mas no estádio adulto tal assimetria pode ser menor (Solon 1980; Veblen 1992). Adicional- mente, as condições e recursos disponíveis em diferentes porções do gradiente vertical de uma floresta estabelecem nichos diferenciados para as plantas (Hubbell \& Foster 1990). Em uma floresta aluvial, onde a umidade do solo deve influenciar de maneira distinta plantas de diferentes estádios de desenvolvimento, o gradiente de umidade pode determinar diferenças estruturais entre os estratos da comunidade.

Neste estudo foram avaliadas a composição florística, a diversidade e a estrutura de três estratos de uma Floresta Ombrófila Mista Aluvial da bacia hidrográfica do rio Iguaçu, no Paraná, e suas relações com a profundidade do lençol freático e com variáveis físico-químicas do solo, buscando-se verificar se a estrutura da floresta pode ser explicada por fatores que atuam diferentemente em plantas de distintos estádios de desenvolvimento.

\section{Material e métodos}

O local de estudo está inserido nos domínios da Floresta Ombrófila Mista, em sua sub-formação Aluvial, no rio Barigüi, bacia hidrográfica do rio Iguaçu, no município de Araucária, Paraná $\left(25^{\circ} 35^{\prime} 12\right.$ "S e $49^{\circ} 20^{\prime} 53,5^{\prime \prime} \mathrm{W}$, a $920 \mathrm{~m}$ de altitude). Nesta região este tipo vegetacional situa-se em terrenos baixos do Holoceno, em solos sazonalmente cobertos pelas águas fluviais, formando charcos temporários (Klein \& Hatschbach 1962). De acordo com a classificação de Koeppen, o clima da região é do tipo $\mathrm{Cfb}$ - temperado, com verões frescos, geadas freqüentes, sem estação seca definida, com médias anuais de temperatura do mês mais quente inferiores a $22{ }^{\circ} \mathrm{C}$ e do mês mais frio inferiores a $18^{\circ} \mathrm{C}$ (IAPAR 1978). A planície aluvionar estudada abrange os solos hidromórficos minerais da ordem dos Gleissolos, inseridos na categoria dos Háplicos e dos Melânicos. A vegetação da região é caracterizada pela Floresta Ombrófila Mista Aluvial em bom estado de conservação, mas em alguns locais descaracterizada, devido à alteração natural do curso do rio Barigüi (Barddal 2004a).

Um fragmento dessa vegetação, com área de aproximadamente $5 \mathrm{ha}$, foi escolhido para este estudo, no interior do qual foi delimitada para amostragem uma área de 0,2 ha, dividida em 20 parcelas de $10 \mathrm{~m} \times 10 \mathrm{~m}$ cada. Para caracterizar a oscilação sazonal do lençol freático, foi instalado, próximo ao centro de cada parcela, um piezômetro por meio do qual a profundidade do lençol freático foi acompanhada mensalmente durante 12 meses. A partir dos dados mensais calcularam-se os valores médios de altura do lençol freático de cada parcela a fim de utilizar nas relações com as distribuições das espécies. A caracterização pedológica de cada unidade 
amostral foi realizada por meio da perfuração do solo com trado e coleta dos horizontes A e C, no centro de cada parcela de $10 \mathrm{~m} \times 10 \mathrm{~m}$ (20 pontos de amostra), ao mesmo tempo em que se fazia sua descrição morfológica. As amostras foram levadas ao laboratório de Solos e Nutrição Florestal da Empresa Brasileira de Pesquisa Agropecuária (EMBRAPA-Florestas) onde, seguindo as normas usuais (EMBRAPA 1979), foram feitas análises químicas e granulométricas.

Entre dezembro/2001 e janeiro/2002, foi feito um levantamento de espécies arbóreas e arbustivas do estrato superior (indivíduos com Perímetro à Altura do Peito PAP $\geq 15 \mathrm{~cm}$, nas 20 parcelas de $10 \mathrm{~m} \times 10 \mathrm{~m}$ ), do estrato intermediário $(\mathrm{PAP}<15 \mathrm{~cm}$, altura $>1,30 \mathrm{~m}$; em 20 sub-parcelas de $5 \mathrm{~m} \times 5 \mathrm{~m}$ sistematicamente alocadas no interior de cada uma das parcelas do estrato superior) e do estrato de regeneração $(0,20 \mathrm{~m}<$ altura $<1,30 \mathrm{~m}$; em quatro sub-parcelas de $1 \mathrm{~m} \times 1 \mathrm{~m}$, em 80 sub-parcelas alocadas no interior das parcelas do estrato superior). Todos os indivíduos arbóreos e arbustivos foram amostrados, sendo anotados a espécie e o diâmetro do colo. Quando necessário, material testemunho foi coletado e preparado de acordo com técnicas usuais de herborização, para posterior determinação. A organização sistemática das famílias foi feita de acordo com APG II - Angiosperm Phylogeny Group versão II (2003) e a verificação dos nomes científicos, conforme Missouri Botanical Garden (1968). Os dados totais foram utilizados para a estimativa da densidade de cada espécie (indivíduos.ha-1) e para cálculos de densidade e índice de diversidade de Shannon (H') (Mueller-Dombois \& Ellenberg 1974), para a comunidade como um todo em cada estrato. Análises florísticas e fitossociológicas dos estratos intermediário e superior foram publicadas, respectivamente, em Barddal 2004a e Barddal 2004b.

Para a comparação florística entre estratos, utilizou-se o Índice de Similaridade de Sørensen (MuellerDombois \& Ellenberg 1974). Para comparação dos índices de diversidade de Shannon, utilizou-se o teste $t$ específico para este índice (Zar 1999). A riqueza de espécies nos três estratos foi comparada através de curva de rarefação, com o programa EstimateS (Cowell 2005). Nesta análise é construída uma curva média de número de espécies em função do número de indivíduos amostrados (valores cumulativos) após 100 aleatorizações dos dados observados, corrigindo eventuais distorções provenientes de tamanhos amostrais distintos nos três estratos.

Visando determinar se o padrão florístico dos diferentes estratos nas parcelas está relacionado com fatores hidrológicos e edáficos, foram relacionados os dados de abundância de indivíduos de cada espécie com a profundidade média anual do lençol freático, fertilidade do solo (porcentagem de argila, soma de bases, quantidade de matéria orgânica e concentração de íons alumínio) além de ter sido incluído nas análises o tipo de solo em cada parcela. Com base nesses dados, procedeu-se, para cada estrato, uma análise de ordenação por DCA (Detrended Correspondence Analysis), através do programa PC-ORD 5.0. Em cada situação foram construídas duas matrizes, a primeira contendo os dados de abundância de espécies e a segunda contendo os dados das variáveis do solo nas parcelas. Como outras análises de correspondência, a DCA ordena espécies e amostras (parcelas) simultaneamente, mas tem a vantagem de ser aplicável em casos onde não há previsibilidade na distribuição das amostras e espécies no espaço (McCune \& Grace 2002). Após a determinação dos dois eixos da análise, a significância dos coeficientes de correlação foi verificada em tabela para diferentes intervalos de confiança (Zar 1999).

\section{Resultados}

No estrato de regeneração foram amostrados 586 indivíduos distribuídos 34 espécies sendo as mais representativas em termos de abundância Allophyllus edulis (245 indivíduos), Myrciaria tenella (65), Myrrhinium atropurpureum (35), Matayba elaeagnoides (27) e Eugenia uniflora (24). No estrato intermediário foram encontrados 918 indivíduos de 39 espécies. A. edulis (327 indivíduos), Daphnopsis racemosa (112), M. tenella (89), E. uniflora (43) e Blepharocalix salicifolius (40) foram as espécies mais abundantes. No estrato superior foram amostrados 507 indivíduos de 29 espécies, sendo Sebastiania commersoniana (326 indivíduos), A. edulis (39), M. atropurpureum (26) e Myrceugenia glaucescens (23) as mais abundantes (Tab. 1 e 2).

Considerando os três estratos juntos, foram amostradas 52 espécies (Tab. 1). As espécies comuns aos três estratos corresponderam a $31 \%$ do total. As porcentagens de espécies exclusivas nos estratos de regeneração, intermediário e superior foram, respectivamente, $10 \%, 19 \%$ e $10 \%$ (Tab. 1). O índice de similaridade florística de Sørensen (S) revelou grande semelhança entre estratos, sendo maior a semelhança entre o estrato de regeneração e o intermediário $(\mathrm{S}=0,71)$, seguido pelos estratos de regeneração e superior $(\mathrm{S}=0,67) \mathrm{e}$, por último, estrato intermediário e superior $(S=0,65)$.

Espécies que eram raras (consideradas as que apresentavam densidade menor que $10 \%$ da densidade total do compartimento) no estrato de regeneração e intermediário também foram raras ou não ocorreram no estrato superior (Tab. 1). Já Dalbergia frutescens, 
Tabela 1. Relação de espécies, respectivas famílias, abreviações e densidades estimadas (indivíduos.ha ${ }^{-1}$ ) nos estratos de regeneração, intermediário e superior de uma Floresta Ombrófila Mista Aluvial no Estado do Paraná, Brasil. (*) espécie de hábito predominantemente arbustivo (**) espécie exótica. (r) espécie rara no referido estrato (densidade $<10 \%$ da densidade total do compartimento).

\begin{tabular}{|c|c|c|c|c|}
\hline \multirow[t]{2}{*}{ Família/Espécie } & \multirow[t]{2}{*}{ Abreviação } & \multicolumn{3}{|c|}{ Estratos } \\
\hline & & Regeneração & Intermediário & Superior \\
\hline \multicolumn{5}{|l|}{ ANARCADIACEAE } \\
\hline Lithraea brasiliensis Marchand & LBRAS & - & - & 30 \\
\hline Schinus terebinthifolius Raddi & STERE & - & - & 90 \\
\hline \multicolumn{5}{|l|}{ AQUIFOLIACEAE } \\
\hline Ilex cf. brevicuspis Reiss. & IBREV & - & $20(\mathrm{r})$ & - \\
\hline \multicolumn{5}{|l|}{ ARECACEAE } \\
\hline Syagrus romanzoffiana (Cham.) Glassman & SROMA & $125(\mathrm{r})$ & $100(\mathrm{r})$ & - \\
\hline \multicolumn{5}{|l|}{ CANELLACEAE } \\
\hline Capsicodendron dinisii (Schwacke) Occhioni & CDINI & - & $120(\mathrm{r})$ & - \\
\hline \multicolumn{5}{|l|}{ CELASTRACEAE } \\
\hline Maytenus ilicifolia (Schrad.) Planch.) & MILIC & 1.750 & 240 & - \\
\hline \multicolumn{5}{|l|}{ ERYTHROXYLACEAE } \\
\hline Erythroxylum myrsinites Mart. & EMYRS & - & $40(r)$ & - \\
\hline \multicolumn{5}{|l|}{ EUPHORBIACEAE } \\
\hline Sebastiania brasiliensis Spreng. & SBRAS & $250(\mathrm{r})$ & 480 & $15(\mathrm{r})$ \\
\hline S. commersoniana (Baillon) Smith \& Downs & SCOMM & 1.625 & 700 & 1.630 \\
\hline \multicolumn{5}{|l|}{ FABACEAE - FABOIDEAE } \\
\hline Dalbergia frutescens (Vell.) Britton & DFRUT & 2.000 & $20(\mathrm{r})$ & $10(\mathrm{r})$ \\
\hline Machaerium paraguariense Hassl. & MPARA & 1.375 & 180 & 50 \\
\hline Xylosma pseudosalzmanii Sleumer & XPSEU & - & $20(\mathrm{r})$ & $5(\mathrm{r})$ \\
\hline \multicolumn{5}{|l|}{ FABACEAE - MIMOSOIDEAE } \\
\hline Inga marginata Willd. & IMARG & - & - & $5(\mathrm{r})$ \\
\hline \multicolumn{5}{|l|}{ ICACINACEAE } \\
\hline Citronela sp. & CITRO & $500(\mathrm{r})$ & - & - \\
\hline \multicolumn{5}{|l|}{ LAURACEAE } \\
\hline Nectandra megapotamica (Spreng.) Mez & NMEGA & $375(r)$ & $40(r)$ & - \\
\hline Ocotea puberula (Rich.) Nees & OPUBE & $375(\mathrm{r})$ & - & - \\
\hline O. pulchella Mart. & OPULC & $375(r)$ & $40(\mathrm{r})$ & - \\
\hline \multicolumn{5}{|l|}{ LOGANIACEAE } \\
\hline Strychnos brasiliensis (Spreng.) Mart. & SBRSS & $250(\mathrm{r})$ & $80(r)$ & - \\
\hline \multicolumn{5}{|l|}{ MELASTOMATACEAE } \\
\hline Miconia cinerascens Miq. (*) & MCINE & - & $20(r)$ & - \\
\hline \multicolumn{5}{|l|}{ MELIACEAE } \\
\hline Trichilia elegans A. Juss. & TELEG & $250(\mathrm{r})$ & $160(\mathrm{r})$ & $5(\mathrm{r})$ \\
\hline \multicolumn{5}{|l|}{ FABACEAE - MIMOSOIDEAE } \\
\hline Inga marginata Willd. & IMARG & - & - & $5(\mathrm{r})$ \\
\hline MYRTACEAE & & & & \\
\hline Blepharocalyx salicifolius (Kunth) Berg. & BSALI & 2.625 & - & 45 \\
\hline Calyptranthes concinna DC. & CCONC & $500(\mathrm{r})$ & $160(\mathrm{r})$ & $5(\mathrm{r})$ \\
\hline Campomanesia xanthocarpa Berg. & CXANT & 1.000 & - & $3(r)$ \\
\hline Eugenia uniflora $\mathrm{L}$. & EUNIF & 3.000 & 860 & $10(r)$ \\
\hline E. uruguayensis Camb. & EURUG & 1.000 & 460 & $5(r)$ \\
\hline Gomidesia palustris (DC.) D. Legr. & GPALU & - & $20(r)$ & - \\
\hline Myrceugenia glaucescens (Camb.) Legr. \& Kaus. & MGLAU & 875 & 440 & 115 \\
\hline Myrcia hatschbachii D. Legr. & MHATS & $125(\mathrm{r})$ & $120(\mathrm{r})$ & - \\
\hline M. laruotteana Camb. & MLARU & - & $40(\mathrm{r})$ & - \\
\hline Myrcia sp. & MYRCI & $125(\mathrm{r})$ & - & - \\
\hline Myrcianthes gigantea (D. Legr.) D. Legr. & MGIGA & $625(\mathrm{r})$ & $80(r)$ & $5(\mathrm{r})$ \\
\hline Myrciaria tenella (DC.) Berg. $(*)$ & MTENE & 8.125 & 1.780 & - \\
\hline Myrrhinium atropurpureum Schott & MARTRO & 4.375 & 560 & 130 \\
\hline Espécie Indeterminada 1 & INDET & $250(\mathrm{r})$ & - & - \\
\hline Espécie Indeterminada 2 & INDEE & $125(\mathrm{r})$ & - & - \\
\hline MYRSINACEAE & & & & \\
\hline Myrsine umbellata Mart. & MUMBE & - & $20(r)$ & - \\
\hline OLEACEAE & & & & \\
\hline Ligustrum vulgare L. (**) & LVULG & $125(\mathrm{r})$ & $20(r)$ & $10(\mathrm{r})$ \\
\hline
\end{tabular}




\begin{tabular}{|c|c|c|c|c|}
\hline \multirow[t]{2}{*}{ Família/Espécie } & \multirow[t]{2}{*}{ Abreviação } & \multicolumn{3}{|c|}{ Estratos } \\
\hline & & Regeneração & Intermediário & Superior \\
\hline \multicolumn{5}{|l|}{ PODOCARPACEAE } \\
\hline Podocarpus lambertii Klotsch ex Endl. & PLAMB & - & $20(\mathrm{r})$ & - \\
\hline \multicolumn{5}{|l|}{ RHAMNACEAE } \\
\hline Hovenia dulcis Thunb. (**) & HDULC & - & - & $15(\mathrm{r})$ \\
\hline Scutia buxifolia Reiss. & SBUXI & 1.125 & 400 & 25 \\
\hline \multicolumn{5}{|l|}{ ROSACEAE } \\
\hline Prunus sellowii Koehne & PSELO & $250(\mathrm{r})$ & - & $5(r)$ \\
\hline \multicolumn{5}{|l|}{ RUBIACEAE } \\
\hline Guettarda uruguensis Cham. \& Schltdl. & GURUG & - & 440 & 55 \\
\hline Rudgea parquioides (Cham.) M. Arg. (*) & RPARQ & - & $40(r)$ & - \\
\hline \multicolumn{5}{|l|}{ SALICACEAE } \\
\hline Casearia decandra Jacquin & CDECA & 1.500 & 260 & $10(\mathrm{r})$ \\
\hline Xylosma pseudosalzmanii Sleumer & XPSEU & - & $20(\mathrm{r})$ & $5(\mathrm{r})$ \\
\hline \multicolumn{5}{|l|}{ SAPINDACEAE } \\
\hline Allophylus edulis (A. St.-Hil.) Radlk. ex Warm. & AEDUL & 30.625 & 6.540 & 195 \\
\hline Cupania vernalis Camb. & CVERN & 1.000 & - & - \\
\hline Matayba elaeagnoides Radlk. & MELAE & 3.375 & 620 & - \\
\hline \multicolumn{5}{|l|}{ PICRAMNIACEAE } \\
\hline Picramnia parvifolia Engler ex Chart. & PPARV & $250(\mathrm{r})$ & $140(\mathrm{r})$ & $15(\mathrm{r})$ \\
\hline \multicolumn{5}{|l|}{ SYMPLOCACEAE } \\
\hline Symplocos uniflora (Pohl.) Benth. & SUNIF & - & $20(\mathrm{r})$ & - \\
\hline \multicolumn{5}{|l|}{ THYMELAEACEAE } \\
\hline Daphnopsis racemosa Griseb & DRACE & 2.857 & 2.240 & - \\
\hline \multicolumn{5}{|l|}{ TILIACEAE } \\
\hline Luehea divaricata Mart. \& Zucc. & LDIVA & - & - & $10(\mathrm{r})$ \\
\hline \multicolumn{5}{|l|}{ VERBENACEAE } \\
\hline Vitex megapotamica (Sprengel) Moldenke & VMEGA & - & $20(\mathrm{r})$ & $5(r)$ \\
\hline Densidade total & & 73.107 & 17.560 & 2.503 \\
\hline
\end{tabular}

Tabela 2. Parâmetros estruturais dos estratos de regeneração $(20 \mathrm{~cm}$ $\leq$ altura $\leq 1,30 \mathrm{~m}$ ), intermediário (perímetro à altura do peito - PAP $<15 \mathrm{~cm}$, altura $>1,30 \mathrm{~m}$ ) e superior (PAP $\geq 15 \mathrm{~cm}$ ) de uma Floresta Ombrófila Mista Aluvial no Estado do Paraná, Brasil.

\begin{tabular}{lccc}
\hline Parâmetro & \multicolumn{3}{c}{ Estratos } \\
\cline { 2 - 4 } & Regeneração & Intermediário & Superior \\
\hline Abundância total & 586 & 918 & 507 \\
$\begin{array}{l}\text { Densidade estimada } \\
\quad \text { (indivíduos.ha }{ }^{-1} \text { ) }\end{array}$ & 73.107 & 17.560 & 2.503 \\
Riqueza & 34 & 39 & 29 \\
H' & 2,36 & 2,49 & 1,59 \\
\hline
\end{tabular}

Campomanesia xanthocarpa, Eugenia uniflora e Eugenia uruguensis, que eram abundantes nos estratos de regeneração e intermediário, mostraram-se raras no estrato superior (Tab. 1).

A partir do estrato de regeneração até o estrato superior, a densidade e o índice de diversidade diminuíram (Tab. 2). Os valores do índice de diversidade de Shannon ( $\left.\mathrm{H}^{\prime}\right)$ diferiram entre os estratos (todos os pares $t>71,13 ; P<0,05)$ sendo maior no estrato intermediário, seguido pelos estratos de regeneração e o superior (Tab. 2). A curva de rarefação (Fig. 1) mostrou que para um mesmo número de indivíduos (500) o número de espécies no estrato de regeneração é maior que os demais estratos, demonstrando que a riqueza diminui gradativamente a partir do estrato de regeneração, para os estratos intermediário e superior.

As DCAs geradas em cada um dos estratos resultaram nos eixos 1 e 2 dos diagramas (Fig. 2). Nem todas as variáveis avaliadas tiveram relação com os eixos interpretados; soma de bases e porcentagem de argila não foram variáveis significativas em nenhuma DCA. Além disso, as variáveis que compõem os eixos 1 e 2 não foram as mesmas nos diferentes estratos (Tab. 3). Para o estrato superior, os eixos 1 e 2 são correlacionados com a quantidade de matéria orgânica e a profundidade do lençol freático (Tab. 3). No estrato superior (Fig 2A) é possível verificar uma distinção de grupos de espécies mais relacionadas com solos ricos em matéria orgânica (por exemplo, Machaerium paraguariensis, Inga marginata, Scutia buxifolia), de espécies relacionadas a solos melhor drenados (Casearia decandra, Hovenia dulcis, Picramia parvifolia) ou ocorrentes em locais de lençol freático mais superficial (Xylosma 


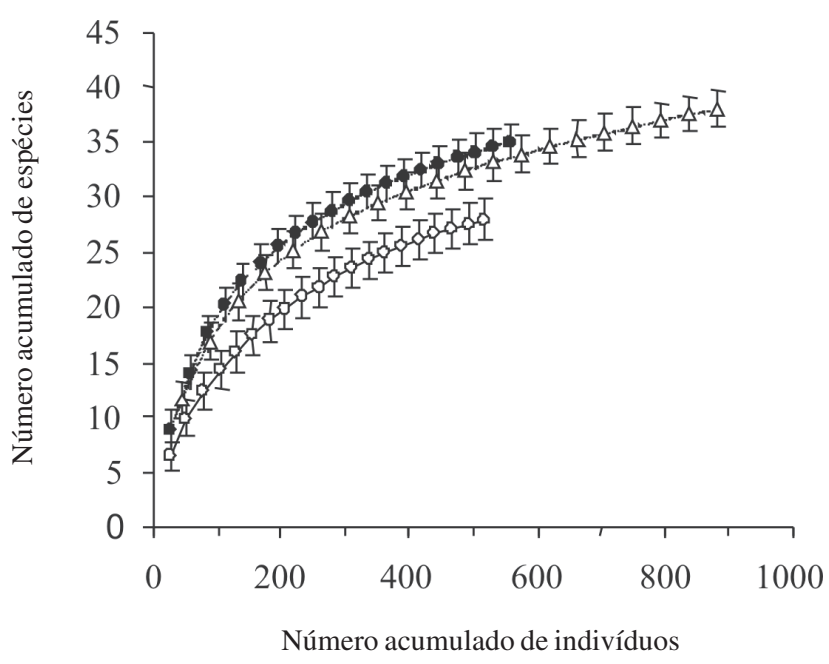

Figura 1. Curva de rarefação para o número de espécies ( \pm 1 desvio padrão) amostradas em 20 parcelas de uma Floresta Ombrófila Mista Aluvial no Estado do Paraná, Brasil. (-o- Estrato superior; -- $\triangle-$-Estrato intermediário; --•-- Estrato de regeneração).

pseudosalzmanii, Luehea divaricata, Sebastiania brasiliensis). No estrato intermediário, o eixo 1 representa a profundidade do lençol freático e o teor de alumínio (Tab 3). Espécies com ocorrência em solos melhor drenados e com maiores teores de alumínio foram, por exemplo, Podocarpus lambertii, Myrceugenia glauscences e C. decandra (Fig 2B). No estrato de regeneração, nenhuma das variáveis apresentou coeficientes de correlação significativos com os eixos da DCA, indicando que as variáveis do solo avaliadas e a profundidade do lençol freático não explicam a distribuição de abundância das espécies entre parcelas.
Tabela 3. Coeficientes de correlação para cada variável, comprimento do gradiente e autovalores para os eixos gerados pelas DCAs dos três estratos de uma Floresta Ombrófila Mista Aluvial no Estado do Paraná, Brasil. $* P<0,05 ; * * P<0,01 ;$ ns $=$ não significativo $(P>0,05)$.

\begin{tabular}{lcc}
\hline Estrato/variável & Eixo 1 & Eixo 2 \\
\hline Estrato superior & & \\
$\quad$ Profundidade do lençol freático & $\mathrm{ns}$ & $0,39^{*}$ \\
$\quad$ Matéria orgânica & $-0,47^{*}$ & $\mathrm{~ns}$ \\
$\quad$ Alumínio & $\mathrm{ns}$ & $\mathrm{ns}$ \\
$\quad$ Comprimento do gradiente & 1,19 & 1,06 \\
$\quad$ Autovalor & 0,17 & 0,09 \\
Estrato intermediário & & \\
$\quad$ Profundidade do lençol freático & $-0,49^{*}$ & $\mathrm{~ns}$ \\
Matéria orgânica & $\mathrm{ns}$ & $\mathrm{ns}$ \\
$\quad$ Alumínio & $-0,62^{* *}$ & $\mathrm{~ns}$ \\
$\quad$ Comprimento do gradiente & 1,95 & 1,57 \\
Autovalor & 0,31 & 0,11 \\
Estrato de regeneração & & \\
$\quad$ Profundidade do lençol freático & $\mathrm{ns}$ & $\mathrm{ns}$ \\
$\quad$ Matéria orgânica & $\mathrm{ns}$ & $\mathrm{ns}$ \\
$\quad$ Alumínio & $\mathrm{ns}$ & $\mathrm{ns}$ \\
$\quad$ Comprimento do gradiente & 1,60 & 1,65 \\
$\quad$ Autovalor & 0,36 & 0,19 \\
\hline
\end{tabular}

\section{Discussão}

A Floresta Ombrófila Mista Aluvial estudada é caracterizada por valores relativamente baixos de diversidade em todos os estratos, o que demonstra o caráter restritivo dos ambientes aluviais (Jacomine 2001; Bianchini et al. 2003). A profundidade do lençol freático, a quantidade de matéria orgânica e os teores de alumínio, que são fatores que geralmente influenciam na ocorrência
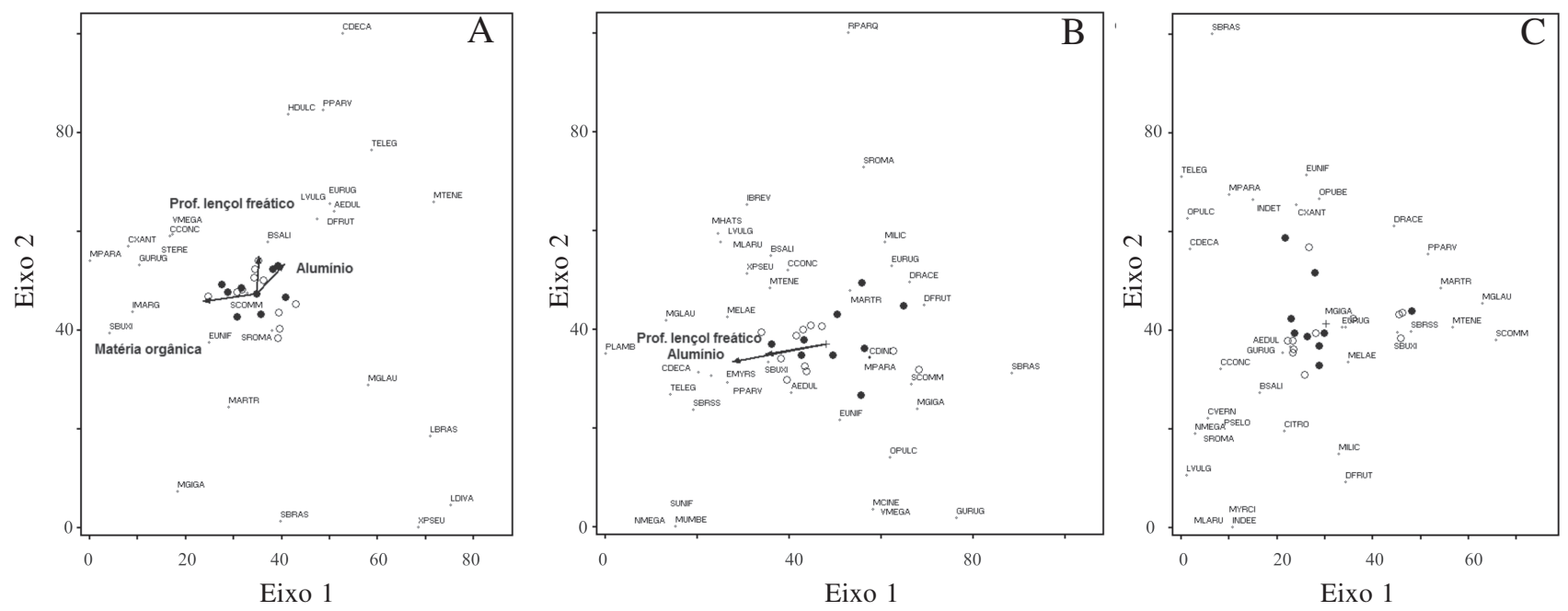

Figura 2. Distribuição das parcelas e espécies de acordo com os eixos 1 e 2 da DCA gerada a partir dos dados de abundância das espécies ocorrentes no estrato superior (A), intermediário (B) e de regeneração (C) em uma Floresta Ombrófila Mista Aluvial no Estado do Paraná, Brasil. Abreviações das espécies de acordo com a Tab. 1 (Classe de solo da parcela: $\bigcirc=$ Gleissolo Háplico; $\bullet$ Gleissolo Melânico). 
das espécies (Ponnamperuma 1984; Lima et al. 2003), tiveram relação com a distribuição das abundâncias das espécies no local de estudo. No entanto, as diferenças estruturais e florísticas entre estratos, somadas aos diferentes níveis de relação das abundâncias das espécies com os fatores edáficos e hídricos, sugerem complexas interações entre a comunidade vegetal e o ambiente.

Nos estratos de regeneração e intermediário, Allophyllus edulis foi a espécie mais abundante, o que é esperado, uma vez que possui ampla distribuição e ocorre associada a planícies aluvionares (Reitz et al. 1978; Reitz 1980; Cervi et al. 1989). Myrciaria tenella foi a segunda espécie mais abundante nos estratos de regeneração e intermediário e é espécie comum em florestas de galeria (Legrand \& Klein 1978). Sebastiania commersoniana, que era menos abundante no estrato de regeneração e intermediário, foi a mais abundante no estrato superior, o que sugere falhas no processo reprodutivo ou então exigências ambientais diferenciadas para plantas de diferentes estádios.

No geral, todos os estratos apresentaram baixa diversidade florística $\left(\mathrm{H}^{\prime} \leq 2,49\right)$ quando comparada, por exemplo, àqueles valores observados em Florestas Ombrófilas Mistas Montanas ( $\mathrm{H}^{\prime}=3,00$, Nascimento et al. 2001; $\mathrm{H}^{\prime}=2,70$, Kozera et al. 2006). Florestas aluviais tendem a apresentar menor diversidade de espécies quando comparadas às florestas situadas em áreas de drenagem livre (Whittaker 1972; Richards 1979; Nebel et al. 2001). As condições resultantes da saturação hídrica do solo em geral levam à dominância de poucas ou de uma única espécie (Richards 1979; Bianchini et al. 2003).

Os estratos apresentaram alta similaridade florística entre si (índices de similaridade superiores a 0,65). No entanto, algumas espécies relativamente abundantes no estrato de regeneração tiveram menos indivíduos no estrato intermediário e deixaram de ocorrer no superior (por exemplo, Maytenus ilicifolia, Myrciaria tenella, Matayba elaeagnoides, Citronela sp.). Por outro lado, espécies que não estavam presentes no estrato de regeneração foram amostradas nos outros estratos (Lithraea brasiliensis, Schinus terebinthifolius, Xylosma pseudosalzmanii, Inga marginata, Hovenia dulcis, Guetterda uruguensis, Luehea divaricata, Vitex megapotamica). É possível que a dinâmica destes ambientes aluvionares tenha resultado em alterações ambientais (por exemplo, na umidade do solo, na disponibilidade de luz, entre outros) que interferiram no estabelecimento de plantas em diferentes momentos, causando as diferenças nas ocorrências das espécies nos estratos. Com isso, houve uma progressiva diminuição da similaridade florística, a partir do estrato de regeneração. No estrato intermediário, a presença de algumas espécies, predominantemente arbustivas
(M. tenella, Rudgea parquioides e Miconia cinerascens), fez com que houvesse uma maior proporção de espécies exclusivas (19\%), diferenciando-se, principalmente, em relação ao estrato superior.

A diversidade e a riqueza foram decrescentes do estrato de regeneração para o superior, demonstrando que, embora um grande número de propágulos possa chegar até a floresta aluvial, fatores relacionados com a qualidade dos microsítios de regeneração limitam o estabelecimento de muitas delas. A diminuição da riqueza de espécies a partir do estrato de regeneração para o superior diverge de resultados previamente encontrados em Florestas de Restinga (Marques 2002), onde a diversidade aumentou a partir do estrato de regeneração. Isto pode indicar que, em florestas aluviais como a estudada, a restrição ao estabelecimento seja forte nas etapas iniciais do desenvolvimento da planta, impondo uma seletividade que resulta em poucas populações de alta abundância no estrato superior. Entre um estrato e outro, existe uma grande diferença em relação às condições ambientais, como a intensidade luminosa, de umidade do ar e de vento (Richards 1979), resultando em nichos distintos para o estabelecimento das plantas (Grubb 1977; Hubbell \& Foster 1990). Desta forma, cada estrato é constituído por um grupo de espécies tolerantes a uma determinada situação.

A distribuição das espécies nas parcelas nos diferentes estratos teve relação com algumas características do solo, sendo profundidade do lençol freático e teores de matéria orgânica e alumínio as mais importantes. Apesar disso, os resultados da DCA mostraram que indivíduos dos diferentes estratos apresentam relações diferenciadas com a profundidade do lençol e os fatores do solo. Tais diferenças devem ser decorrentes do porte distinto das plantas (principalmente profundidade da raiz) que faz com que a forma de absorção de água e nutrientes e, conseqüentemente, as exigências metabólicas, sejam diferenciadas. Isto foi mais evidente para a distribuição dos indivíduos do estrato de regeneração, que não apresentou fortes relações com as variáveis avaliadas. As plantas deste estrato, pequenas e com sistemas radiciais pouco desenvolvidos, devem sofrer pouca influência da profundidade do lençol freático (que influenciou os outros dois estratos), sendo sua distribuição, neste estádio, mais provavelmente decorrente de fatores biológicos (e.g. dispersão, competição) ou de outros fatores ambientais não analisados neste estudo, como, por exemplo, a disponibilidade de luz. Em longo prazo, à medida que as plantas crescem, a pressão de seleção oriunda do ambiente, especialmente da profundidade do lençol freático, levaria à eliminação de espécies que, embora tenham sido amostradas no estrato de regeneração, não seriam recrutadas para os estratos 
intermediário e superior.

Lençol freático pouco profundo restringe a ocorrência de espécies em ambientes aluviais (Joly 1991; Lobo \& Joly 2001; Medri et al. 2002) e este fator teve forte influência na ocorrência de espécies tolerantes (Sebastiania brasiliensis, Xylosma pseudosalzmanii, Luehea divaricata) e menos tolerantes (Casearia decandra, Hovenia dulcis, Picramnia parvifolia) à saturação hídrica do solo. Ao excesso de íons alumínio é usualmente atribuída toxicidade para muitas espécies de plantas (Jacomine 2001; Gonçalves et al. 2003; Haridasan \& Araújo 2005) o que pode ter restringido a ocorrência de algumas espécies intolerantes em algumas parcelas, não interferindo na distribuição de espécies aparentemente mais tolerantes, tais como Podocarpus lamberti. No entanto, é importante ressaltar que existe uma forte covariância entre concentração de alumínio e profundidade do lençol freático, o que muitas vezes dificulta a interpretação da distribuição das espécies em função deste fator edáfico.

Altos teores de matéria orgânica aumentam a fertilidade do solo, o que é essencial para o metabolismo vegetal (Malavolta 2006). A maior parte das espécies do estrato superior esteve positivamente relacionada com este fator e apenas algumas delas (como, por exemplo, Myrciaria tenella e Allophylus edulis) parecem não necessitar de solos férteis para seu desenvolvimento.

Embora as comunidades ocorrentes em ambientes ripários sejam geralmente interpretadas apenas à luz da distribuição de indivíduos adultos (que formam o estrato superior) e em relação ao excesso de água no solo, os resultados encontrados na Floresta Ombrófila Mista Aluvial aqui estudada demonstram que plantas de outros estádios de desenvolvimento (estratos intermediário e de regeneração) e outros fatores do solo (em especial os fatores químicos) devem também ser considerados. Portanto, a estrutura e riqueza destas comunidades resultam de diferentes níveis de interação ao longo do ciclo de vida dos indivíduos.

\section{Agradecimentos}

A Gustavo Ribas Curcio, pela orientação nas coletas e análises do solo em laboratório; à Giselda Durigan e a dois revisores anônimos, pelas sugestões ao manuscrito; ao $\mathrm{CNPq}$, pela bolsa concedida à primeira autora; à Refinaria Presidente Getúlio Vargas (REPAR), pelo apoio financeiro ao projeto "Diagnóstico e monitoramento da cobertura vegetal da área de influência direta e indireta do vazamento de óleo da REPAR, Araucária, PR", no qual este estudo esteve inserido; à Capes pelo apoio financeiro (bolsa pós-doc) à Márcia C.M. Marques, quando da finalização deste artigo.

\section{Referências bibliográficas}

Angisosperm Phylogeny Group II. 2003. An update of the Angiosperm phylogeny group classification for the ordens and families of flowering plants: APG II. Annals of the Botanical Journal of the Linnean Society 141: 399-436.

Barddal, M.L.; Roderjan, C.V.; Galvão, F. \& Curcio, G.R. 2004. Fitossociologia do sub-bosque de uma Floresta Ombrófila Mista Aluvial, no município de Araucária, PR. Ciência Florestal 14: $35-45$.

Barddal, M.L.; Roderjan, C.V.; Galvão, F. \& Curcio, G.R. 2004. Caracterização florística e fitossociológica de um trecho sazonalmente inundável de floresta aluvial, em Araucária, PR. Ciência Florestal 14: $37-50$.

Bianchini, E.; Popolo, R.S.; Dias, M.C. \& Pimenta, J.A. 2003. Diversidade e estrutura de espécies arbóreas em área alagável do município de Londrina, Sul do Brasil. Acta Botanica Brasilica 17: 405-419.

Cervi, A.C.; Paciornik, E.F.; Vieira, R.F. \& Marques, L.C. 1989. Espécies vegetais de um remanescente de Floresta de Araucária (Curitiba, Brasil): Estudo preliminar I. Acta Biológica Paranaense 18: 73-114.

Connel, J.H.; Tracey, J.G. \& Webb, L.J. 1984. Compensatory recruitment, growth, and mortality as factors maintaining rain forest tree diversity. Ecological Monographs 54: 141-164.

Cowell, R.K. 2005. EstimateS: statistical estimation of species richness and shared species from samples. Version 7.5. Guia do usuário e aplicação disponível em: http://purl.oclc.org/estimates.

Crawley, M.J. 1997. Plant Ecology. $2^{\text {nd }}$ ed. Oxford, Blackwell Scientific Publishing.

Curcio, G.R.; Galvão, F.; Bonnet, A.; Barddal, M.L. \& Dedecek, R.A. 2007. A floresta fluvial em dois compartimentos do rio Iguaçu, Paraná, Brasil. Floresta 37: 125-147.

Dalanesi, P.E., Oliveira Filho A.T. \& Fontes, M.A.L. 2004. Flora e estrutura do componente arbóreo da floresta do Parque Ecológico Quedas do Rio Bonito, Lavras, MG e correlações entre a distribuição das espécies e variáveis ambientais. Acta Botanica Brasilica 18: 737-757.

EMBRAPA - CNPF. 1979. Manual de métodos de análise de solo. Brasília, Serviço de produção da informação. Rio de Janeiro, CNPS

Gonçalves, J.L.M.; Nogueira, L.R. \& Ducatti, F. Recuperação de solos degradados. Pp. 111-163. In: Kageyama, P.Y.; Oliveira, R.E.; Moraes, L.F.D.; Engel, V.L. \& Gandara, F.B. 2003. Restauração Ecológica de Ecossitemas Naturais. FEPAF.

Grubb, P.J. 1977. The maintenance of species-richness in plant communities: The importance of the regeneration niche. Biological Review 52: 107-145.

Haridasan, M. \& Araújo, G.M. 2005. Perfil nutricional de espécies lenhosas de duas florestas semidecíduas em Uberlândia, MG. Revista Brasileira de Botânica 28: 295-303.

Hubbell, S.P. \& Foster, R.B. 1990. The fate of juvenile trees in a Neotropical forest: implications for the natural maintenance of tropical tree diversity. In: Bawa, K.S. and Hadley, M. Reproductive Ecology of Tropical Forest Plants. Main and the Biosphere Series. Paris, The Parthenon Publishing Group. UNESCO.

IAPAR. 1978. Cartas climáticas básicas do Estado do Paraná. Londrina.

Ivanauskaus, N.M.; Rodrigues, R.R. \& Nave, A.G. 1997. Aspectos ecológicos de um trecho da floresta de brejo de Itatinga, SP: florística, fitossociologia e seletividade de espécies. Revista Brasileira de Botânica 20: 139-153.

Jacomine, P.K.T. 2001. Solos sob Matas Ciliares. Pp. 27-32. In: R.R. Rodrigues \& H.F. Leitão Filho (eds.). Matas ciliares: conservação e recuperação. $2^{a}$ ed. São Paulo, EDUSP e FAPESP.

Joly, C.A. 1991. Flooding tolerance in tropical trees. Pp. 23-34. In: M.B. Jackson; D.D. Davies \& H. Lambers (eds.). Plant life under oxygen deprivation: ecology, physiology and biochemistry. SBP Academic Publishing, The Hague.

Joly, C.A. 1996. The role of oxygen diffusion to the root system on the flooding tolerance of Brazilian trees. Revista Brasileira de Biologia 56: 375-382. 
Klein, R.M. \& Hatschbach, G. 1962. Fitofisionomia e notas sobre a vegetação para acompanhar a planta fitogeográfica do município de Curitiba e arredores. Boletim da Universidade Federal do Paraná. Geografia Física 4: 1-30.

Kotchetkoff-Henriques, O.; Joly, C.A. \& Bernacci, L.C. 2005. Relação entre solo e a composição florística de remanescente de vegetação natural no município de Ribeirão Preto, SP. Revista Brasileira de Botânica 28: 541-562.

Kozera, C.; Dittrich, V.A.O. \& Silva, S.M. 2006. Fitossociologia do componente arbóreo de um fragmento de Floresta Ombrófila Mista Montana, Curitiba, PR, BR. Floresta 36: 225-237

Legrand, C.D. \& Klein, R.M. 1978. As plantas mirt: mirtáceas. Flora ilustrada Catarinense. Itajaí, Santa Catarina.

Lima, J.A.S.; Meneguelli, N.A.; Gazel Filho, A.B. \& Pérez, D.V. 2003. Agrupamento de espécies arbóreas de uma floresta tropical por características de solo. Pesquisa Agropecuária Brasileira 38: 109-116.

Lobo, P.C. \& Joly, C.A. 2001. Aspectos Ecofisiológicos da Vegetação de Mata Ciliar do Sudeste do Brasil. Pp. 143-157. In: R.R. Rodrigues \& H.F. Leitão Filho (eds.). Matas ciliares: conservação e recuperação. 2 a ed. São Paulo, EDUSP e FAPESP.

Malavolta, E. 2006. Manual de nutrição mineral de plantas. Ed. Ceres.

Marques, M.C.M. 2002. Dinâmica da dispersão de sementes e regeneração de plantas da Planície Litorânea da Ilha do Mel, PR. Tese de doutorado (Biologia Vegetal). Campinas, Universidade Estadual de Campinas.

Marques, M.C.M.; Silva, S.M. \& Salino, A. 2003. Florística e estrutura do componente arbustivo-arbóreo de uma floresta higrófila da bacia do rio Jacaré-Pepira, SP, Brasil. Acta Botanica Brasilica 17: 495-506

McCune, B. \& Grace, J.B. 2002. Analysis of ecological communities. MjM Software Design.

Medri, M.E.; Bianchini, E.; Pimenta, J.A.; Colli, S. \& Müller, C. 2002. Estudos sobre tolerância ao alagamento em espécies arbóreas nativas da bacia do rio Tibagi. Pp. 133-172. In: M. Medri; E. Bianchini; O.A. Shibatta \& J.A. Pimenta. A bacia do rio Tibagi. Londrina.

Missouri Botanical Garden. 1968. Vascular Tropicos (VAST) nomenclatural database and associated authority files. Disponível em: http://www.mobot.org/w3t/search/vast.html.

Mueller-Dombois, D. \& Ellenberg. 1974. Aims and Methods of Vegetation Ecology. New York, J. Wiley \& Sons.
Nascimento, A.R.T.; Longhi, S.J. \& Brena, D. 2001. Estrutura e padrões de distribuição espacial de espécies arbóreas em uma amostra de Floresta Ombrófila Mista em Nova Prata, RS. Ciência Florestal 11: 105-119.

Nebel, G.; Kvist, L.P.; Vanclay, J.K.; Christensen, H.; Freitas, L. \& Ruíz, J. 2001. Structure and floristic composition of flood plain forests in the Peruvian Amazon. I. Overstorey. Forest Ecology and Management 150: 27-57.

Ponnamperuma, F.N. 1984. Effects of flooding on soil. Pp. 10-46. In: T.T. Kozlowski. Flooding and plant growth. London, Academic Press.

Reitz, R; Klein, R.M. \& Reis, A. 1978. Projeto Madeira do Rio Grande do Sul. Porto Alegre, Editora CORAG.

Reitz, R. 1980. As plantas sapi: Sapindáceas. Flora ilustrada Catarinense. Itajaí, Santa Catarina.

Richards, M.A. 1979. The Tropical Rain Forest: an ecological study. London, Cambridge, Cambridge University Press.

Roderjan, C.V.; Galvão, F.; Kuniyoshi, Y.S. \& Hatschbach, G.G. 2002. As unidades fitogeográficas do Estado do Paraná. Ciência \& Ambiente. Fitogeografia do Sul da América 24 (75:92).

Salo, J.S. \& Kalliola, R.J. 1991. River Dynamics and natural forest regeneration in the Peruvian Amazon. In: A. Gómez-Pompa; T.C. Whitmore \& M. Hadley. Rain Forest Regeneration And Management. Paris, The Parthenon Publishing Group. Main And The Biosphere. Series v. 6.

Solon, M.E. 1980. Dinâmica de populações. São Paulo, Editora Pedagógica e Universitária Ltda - EPU. Temas de Biologia 3: 1-78.

Torezan, J.M.D. \& Silveira, M. 2002. Fatores ambientais, diversidade e similaridade em florestas da bacia do rio Tibagi. Pp. 125-131. In: M. Medri; E. Bianchini; O.A. Shibatta J.A. \& Pimenta. A bacia do rio Tibagi. Londrina.

Troppmair, H. \& Machado, M.L.A. 1974. Variação da estrutura da mata galeria na bacia do rio Corumbataí (SP) em relação à água do solo, do tipo de margem e do traçado do rio. Biogeografia 8: 1-128.

Veblen, T.T. 1992. Regeneration dynamics. Pp. 152-187. In: D.C. Glenn-Lewin; R.K. Peet \& T.T. Veblen (eds.). Plant Sucession: Theory and prediction. London, Chapman and Hall.

Veloso, H.P.; Rangel Filho, A.L.R. \& Lima, J.C.A. 1991. Classificação da vegetação brasileira, adaptada a um sistema universal. Rio de Janeiro, Fundação Instituto Brasileiro de Geografia e Estatística - IBGE.

Whittaker, R.H. 1972. Evolution and measurement of species diversity. Taxon 21: 213-251.

Zar, J.H. 1999. Biostatistical Analysis. $4^{\text {th }}$ ed. New Jersey, Prentice Hall

Versão eletrônica do artigo em www.scielo.br/abb e http://www.botanica.org.br/acta/ojs 